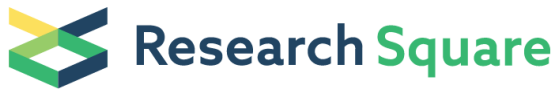 \\ Preprints are preliminary reports that have not undergone peer review. \\ They should not be considered conclusive, used to inform clinical practice, or referenced by the media as validated information.
}

\section{Fine Mapping and Validation of a Major QTL for Grain Weight on Chromosome 5B in Bread Wheat}

\section{Dehui Zhao}

Chinese Academy of Agricultural Sciences Institute of Crop Sciences of Agricultural Sciences (CAAS)

Li Yang

Institute of Crop Sciences, National Wheat Improvement Centre, Chinese Academy of Agricultural Sciences (CAAS)

\section{Dan Liu}

Institute of Crop Sciences, National Wheat Improvement Centre, Chinese Academy of Agricultural Sciences (CAAS)

Jianqi Zeng

Institute of Crop Sciences, National Wehat Improvement Centre, Chinese Academy of Agricultural Sciences (CAAS)

\section{Shuanghe Cao}

Institute of Crop Sceneces, National Wheat Improvement Centre, Chinese Academy of Agricultural Sciences (CAAS)

\section{Xianchun Xia} Institute of Crop Sciences, National Wheat Improvement Centre, Chinese Academy of Agricultural Sciences (CAAS)

Jun Yan

Insitute of Cotton Research, CAAS

\section{Xiyue Song}

College of Agronomy, Northwest A \& F University

\section{Zhonghu He}

Institute of Crop Sciences, National Wheat Improvement Centre, Chinese Academy of Agricultural Sciences (CAAS); CIMMYT-China Office, c/o CAAS

Yong Zhang ( $\sim$ zhangyong05@caas.cn )

Chinese Academy of Agricultural Sciences Institute of Crop Sciences https://orcid.org/0000-0002-3669-3908

\section{Research Article}

Keywords: Thousand grain weight, RIL population, molecular marker for improving grain yield, wheat

Posted Date: May 10th, 2021

DOl: https://doi.org/10.21203/rs.3.rs-495159/v1

License: @ (1) This work is licensed under a Creative Commons Attribution 4.0 International License. Read Full License

Version of Record: A version of this preprint was published at Theoretical and Applied Genetics on July 29th, 2021. See the published version at https://doi.org/10.1007/s00122-021-03925-9. 


\section{Abstract}

Thousand grain weight (TGW), determined by grain length and width, is an important yield component in wheat; understanding of the underlying genes and molecular mechanisms remains limited. A stable QTL QTgw.caas-5B for TGW was identified previously in a RIL population developed from a cross between Zhongmai 871 (ZM871) and a sister line Zhongmai 895 (ZM895), and the aim of this study was to perform fine mapping and validate the genetic effect of the QTL. It was delimited to an interval of approximately $2.0 \mathrm{Mb}$ flanked by markers Kasp_5B29 and Kasp_5B31 (49.6 Mb to $51.6 \mathrm{Mb})$ using 12 heterozygous recombinant plants obtained by selfing a residual $B_{1} F_{4}$ line selected from the $Z M 871 / Z M 895 / / Z M 871$ population. A candidate gene was predicted following sequencing and differential expression analyses. Marker Kasp_5B_Tgw based on a SNP in TraesCS5B02G044800, the QTgw.caas-5B candidate, was developed and validated in a diversity panel of 166 cultivars. The precise mapping of QTgw.caas-5B laid a foundation for cloning of a predicted causal gene and provides a molecular marker for improving grain yield in wheat.

\section{Introduction}

Wheat is one of the most important food crops in the world, providing approximately $20 \%$ of the calories and $25 \%$ of the protein for humans (FAO 2017, http://www.fao.org/faostat/en/). Although significant progress has already been made on yield improvement during the last 60 years, with a genetic gain of 0.7-1.0\% annually (Fischer and Edmeades 2010; Gao et al. 2017), it is estimated that yield must still increase by more than $60 \%$ to meet predicted growth in the world population by 2050 , despite the restricting effects of climate change and the declining area of available land due to urbanization and degradation (Langridge 2013). Improved yield potential is therefore still a major breeding objective. Identification and mining of genetic loci for grain yield will provide genetic resources and tools to improve yield potential.

Yield is a complex quantitative trait determined by thousand grain weight (TGW), grain number per spike and spike number per unit area, with TGW having the highest heritability among three components (Simmonds et al. 2014, 2016; Chen et al. 2020; Yang et al. 2020). TGW is determined by grain size and grain filling characteristics. Grain size can be divided into components grain length (GL), grain width (GW) and grain thickness (Kuchel et al. 2007; Simmons et al. 2014, Yang et al. 2020). Numerous genes for grain weight have been cloned in rice (Li and Li 2016; Li. 2019b) and many genes associated with grain weight in wheat were cloned by comparative genomics (Su et al. 2011; Zhang et al. 2012; Dong et al. 2014; Zhang et al. 2014; Jiang et al. 2015; Wang et al. 2015; Yue et al. 2015; Ma et al. 2016; Wang et al. 2016; Hanif et al. 2016; Hu et al. 2016; Simmonds et al. 2016; Sajjad et al. 2017; Yang et al. 2019; Cao et al. 2020). A large number of quantitative trait loci (QTL) for TGW, GL and GW have also been identified (Huang et al. 2003; Quarrie et al. 2005; Prashant et al. 2012; Cui et al. 2014; Wu et al. 2015; Sun et al. 2017; Cabral et al. 2018; Guan et al; 2018; Ma et al. 2018; Su et al. 2018; Zhai et al; 2018; Yang et al. 2020). However, few TGW genes have been isolated by map-based cloning due to the complexity of the wheat genome. With the current availability of genotyping arrays and release of wheat reference genome sequences (IWGSC, 2018; https://urgi.versailles.inra.fr/blast_iwgsc/) fine mapping of major QTL for TGW has been achieved using near-isogenic lines (NILs) and residual heterozygosity (Brinton et al. 2017; Guan et al. 2019; Xu et al 2019; Chen et al. 2020).

In a previous study (Yang et al 2020) four QTL for TGW-related traits were identified on chromosomes 1AL, 2BS, 3AL and 5B. QTgw.caas-5B, detected across all of ten environments, explaining $5.7-17.1 \%$ of the phenotypic variances, was mapped to a $11.6 \mathrm{cM}$ region between markers Kasp_5B5 and Kasp_5B12, extending from 45.3 to $394.2 \mathrm{Mb}$ in chromosome 5B based on the Chinese Spring reference genome (IWGSC, 2018; https://urgi.versailles.inra.fr/blast_iwgsc/). The present study was to precisely map QTgw.caas-5B by analysis of a residual heterozygous plant, predict one or more candidate genes and develop tightly linked KASP markers for accurate marker-assisted selection in breeding and research.

\section{Materials And Methods}

\section{Plant materials}

Zhongmai 895 (hereafter ZM895) with a current production area around 0.7 million ha annually is a leading cultivar in the Yellow and Huai River Valleys Winter Wheat Region of China. ZM895 and Zhongmai 871 (hereafter ZM871), developed by pedigree selection and bulked as fixed lines at $F_{5}$, are sister lines that can be traced back to a single $F_{2}$ plant of cross Zhoumai $16 /$ Liken 4 . The detailed information of ZM895 and ZM871 was described in a previous study (Yang et al, 2020). One residual heterozygous line (L2925) within the marker interval of QTgw.caas-5B was selected from $\mathrm{BC}_{1} \mathrm{~F}_{6}$ generation of the ZM871/ZM895//ZM871 population (Fig. 1a, 
Fig. 1b). A heterozygous recombinant plant (HRL2925) from L2925 was self-pollinated, and 12 heterozygous recombinant plants (designated RL1 to RL12) and 119 homozygous plants with 57 having 5B+ alleles (ZM895 genotype) and 62 having 5B- (ZM871 genotype) were selected from the selfed progenies by markers flanking $Q T g w . c a a s-5 B$, in which two groups of homozygous plants were used for a preliminary evaluation of the phenotypic effects of QTgw.caas-5B on TGW, GL and GW (Fig. 1c, Fig. 1d). Twenty 5B+ near-isogenic lines (NILs) and 20 5B- generated from two kinds of homozygous plants, respectively, were used to measure TGW, GL and GW at different grain developmental stages and for RNA-sequencing. In addition, 52 to 77 NILs from each of RL1 to RL12 were identified using QTgw.caas-5B-flanking markers to narrow the region of candidate genes (Fig. 1d, Table 2). A diverse panel of 166 cultivars (Li. 2019a) was used to validate the effects of QTgw.caas-5B.

\section{Field trials and trait measurement}

The progeny from HRL2925 were sown in ten $3.0 \mathrm{~m}$ rows spaced $0.3 \mathrm{~m}$ apart with 30 seeds per row at Xinxiang (Henan province) during the 2017-2018 cropping season. Twenty homozygous NILs with 5B+ and 20 with 5B- selected from 119 selfed homozygous plants of HRL2925 were evaluated at Xinxiang and Anyang (Henan province), during the 2018-2019 cropping season. The lines were grown as plots in randomized complete blocks with three replications. Each plot comprised two $1.0 \mathrm{~m}$ rows spaced $0.3 \mathrm{~m}$ apart, with 30 seeds per row. The NILs from each of RL1 to RL12 were sown in plots of seven $3.0 \mathrm{~m}$ rows spaced $0.3 \mathrm{~m}$ apart with 30 seeds per row at Xinxiang (Henan province) during the 2018-2019 cropping season. The panel of 166 cultivars was sown in three $1.5 \mathrm{~m}$ rows spaced $0.2 \mathrm{~m}$ apart in 50 seeds each row with three replications at Suixi (Anhui province) during the 2012-2013, 2013-2014 and 2014-2015 cropping seasons, at Anyang (Henan province) during the 2012-2013 and 2013-2014 cropping seasons, and at Shijiazhuang (Hebei province) during the 2014-2015 cropping season.

Wanshen SC-G seed detector (Hangzhou Wanshen Detection Technology Co., Ltd) was used to record TGW, GL and GW. Thirty random spikes were harvested from each plot of all 20 homozygous individuals in the contrasting $5 \mathrm{~B}+$ and $5 \mathrm{~B}-$ groups to measure TGW, GL and GW. The same parameters were measured on the 119 homozygous plants from HRL2925 were measured on grains from 6-10 spikes of each plant and 52 to 77 homozygous progenies from each of RL1 to RL12. For the diversity panel TGW was determined by weighting 500 grains, and GL and GW were measured on 20 random grains from each plot to obtain mean length and width values, respectively (Li et al. 2019b).

\section{Grain sampling}

Main stems at anthesis were marked with red tags at 09:00-11:00 am every day. Ten grains from outer florets of five spikelets in the middle regions of tagged spikes were sampled at 09:00-11:00 am at 4, 8, 12, 16, 20, 25 and 30 days post-anthesis (DPA). At each time point 200 grains were sampled (20 spikes $\times 10$ grains) from each of the 40 homozygous NILs. GL and GW were measured using image analysis software (Image-Pro Plus 6.0, http://www.mediacy.com/) after scanning the 200 grain samples placed on a scanner panel with grain creases placed downwards. Following measurement, the grains were dried in an oven at $135^{\circ} \mathrm{C}$ for 15 min and then at $65^{\circ} \mathrm{C}$ until a constant weight. The TGW of the dried grain samples harvested at various DPA was determined Three biological replications were performed for each time point.

\section{RNA and DNA extraction and RNA-sequencing}

Two grains sampled from the outer florets of spikelets in the middle of tagged spikes of main stems at 4, 8, 12, 16, 20 and 25 DPA were snap frozen in liquid nitrogen and stored at $-80^{\circ} \mathrm{C}$. For 20 homozygous NILs with 5B+ and 20 with 5B- selected from 119 selfed homozygous plants of HRL2925 at every time point, 40 grains were sampled (20 spikes $\times 2$ grains) with three biological replications.

Total RNA was isolated using the TRIzol protocol (Invitrogen, Carlsbad, CA, USA). After quality testing, a single RNA library for each sample was constructed, and the library preparations were sequenced on an Illumina Hiseq platform with 250-300 bp paired-end reads at Novegene Bioinformatics Technology in Beijing (http://www.novogene.com/). Differential expression analysis of 5B+ and $5 B$ - genotypes was performed with three biological replications at each stage using the DESeq2 R package (1.10.1), which provides the statistical routines for determining differential expression in digital gene expression data using a model based on a negative binomial distribution. The resulting $P$-value was adjusted using the Benjamini and Hochberg's approach for controlling false discovery rate. Genes with an adjusted $P<0.05$ determined by DESeq 2 were considered as differentially expressed. Genomic DNA was extracted from young leaves of experimental lines using the CTAB method.

\section{Whole genome resequencing}


Quanified DNA samples of ZM871 and ZM895 were randomly fragmented by Covaris and the fragments were collected by magnetic beads. Ligation products with end-repair and addition of 3 ' adenine DNA fragments were cycled and amplified by linear isothermal Rolling-Circle Replication and DNA NanoBall technology. Sequencing of these DNA libraries was performed by the BGISEQ sequencing platform at Shenzhen Huada Gene Technology (Shenzhen, https://www.genomics.cn/). The remaining high-quality paired-end reads following filtering of a high proportion of adaptor and low-quality reads in the raw data were mapped to the Chinese Spring reference genome (IWGSC, https://urgi.versailles.inra.fr/blast_iwgsc/) using the Burrows-Wheeler Aligner Tool. SNPs and small Insertion/Deletions (InDels) were detected by GATK (https://www.broadinstitute.org/gatk/) with filtration parameters of "QD $<2.0$ || FS $>60.0|| \mathrm{MQ}<40.0$ || MQRankSum <-12.5 || ReadPosRankSum <-8.0" for SNP calling and "QD < 2.0 || FS > 200.0 || SOR > 10.0 || MQRankSum <-12.5 || ReadPosRankSum <-8.0" for InDel calling.

Array-based SNP markers or SNPs from RNA-sequencing and whole genome resequencing upstream of the physical location of the Kasp_5B2 locus and between Kasp_5B2 and Kasp_5B6 were converted to Kompetitive Allele Specific PCR (KASP) markers for fine mapping of QTgw.caas-5B. Allele-specific and common reverse primers for each KASP marker were designed using PolyMarker (http://www.polymarker.info/).

\section{Statistical Analysis}

Phenotypic differences between the allelic pairs in progeny tests were determined by Student's t-tests with SAS 9.2 software (SAS Institute Inc, Cary, NC, USA).

\section{Results}

\section{Generation of fine mapping population using residual heterozygous lines}

A heterozygous line $L 2925$ screened from a BC ${ }_{1} F_{6}$ population of the ZM871/ZM895//ZM871 had homozygous background across the mapping interval spanning QTgw.caas-5B (Fig. 1a, Fig. 1b; Yang et al. 2020). One recombinant plant (HRL2925) from L2925 was self-pollinated and generated 12 heterozygous recombinant plants (Fig. 1c, Fig. 1d, Table S1). The heterozygous interval was flanked by SNP markers $A X-110372788$ (24450322) and $A X-95631395$ (53662687) from $24.5 \mathrm{Mb}$ to $53.7 \mathrm{Mb}$ in the HRL2925 based on the wheat 50K SNP array developed in collaboration by the Chinese Academy of Agricultural Sciences and Capital-Bio, Beijing, China (https://www.capitalbiotech.com/). The genetic similarity between homozygous progenies for 5B+ and 5B- was more than $99 \%$ according to 50K SNP array data, indicating that the segregating progenies from the HRL2925 were suitable for fine mapping (Table S2). Within each family of selfed progenies from 12 recombinant plants, homozygous non-recombinant plants, namely 5B+NILs and 5B- NILs, were genotyped with markers according to heterozygous interval and phenotypes evaluated for fine mapping.

\section{Phenotypic validation of NILs for QTgw.caas-5B}

After a progeny test, a significant difference in TGW was detected between the genotypes 5B+ with 57 plants and $5 \mathrm{~B}-$ with 62 plants from selfing progenies of HRL2925 (Fig. 2). In order to improve the accuracy of phenotypic identification of recombinants, 20 homozygous NILs with $5 \mathrm{~B}+$ and 20 with $5 \mathrm{~B}$ - generated from two kinds of homozygous plants were evaluated at Xinxiang and Anyang (Henan province) to verify the effects of QTgw.caas-5B. Student's t-tests indicated significantly $(P<0.05)$ higher TGW and GL in 5B+ lines than their contrasting 5B- lines (Fig. 3). These demonstrated that the ZM895 allele at QTgW.caas-5B had a positive effect on TGW.

To further analyze the genetic effect of $Q T g w . c a a s-5 B$, the dynamic change of grain weight and size at different developmental stages between the above NILs 5B+ and 5B- was investigated. Twenty NILs with $5 B+$ and 20 with $5 B$ - were used to determine the differences on grain morphometric parameters. Student's $t$-tests indicated significantly $(P<0.05)$ higher GL in $5 B+N I L s$ than those in 5B-from the 12 DPA, while 5B+ NILs also had significantly $(P<0.05)$ higher TGW than those of 5B-from the 25 DPA (Table 1$)$. No significant differences were observed in $\mathrm{GW}$ between the $5 \mathrm{~B}+$ and $5 \mathrm{~B}$ - genotypes at all the developmental stages. This suggests that the increased TGW is attributed to the increased grain length in the $5 B+$ genotypes.

\section{Fine mapping of $Q T g w . c a a s-5 B$}

For fine mapping of QTgw.caas-5B, eight new markers between Kasp_5B2 and Kasp_5B6 were developed from 660K, 50K SNP arrays and resequencing data (Table S1, Fig. 1d). Twelve heterozygous recombinant plant (RL1 to RL12) identified from HRL2925 using 13 
markers (Fig. 1d) were analysed for fine mapping of QTgw.caas-5B. After progeny tests significant differences in TGW were detected between 5B+ and 5B- genotypes within RL1, RL6, RL7 and RL12 $(P<0.05)$, whereas there were no significant differences within the other NILs from RL2 to RL5 and RL8 to RL11 (Table 2, Fig. 1d). QTgw.caas-5B was delimited to an interval of approximately $2.0 \mathrm{Mb}$ flanked by markers Kasp_5B29 and Kasp_5B31 (49.6-51.6 Mb), with 17 high-confidence genes based on gene annotations for the Chinese Spring reference genome (IWGSC, https://urgi.versailles.inra.fr/blast_iwgsc/).

\section{Prediction of candidate genes for QTgw.caas-5B}

Based on resequencing data for the parents, SNPs or InDels were found in the coding or intron regions of TraesCS5B02G044500, TraesCS5B02G044600, TraesCS5B02G044700, TraesCS5B02G044900, TraesCS5B02G045500, TraesCS5B02G045800,

TraesCS5B02G045900 and TraesCS5B02G046000, whereas another nine high-confidence genes lacked sequence polymorphisms between the two parents.

The results of RNA-seq indicated that only TraesCS5B02G044800 among the 17 high-confidence genes in the 49.6-51.6 Mb region on chromosome $5 \mathrm{~B}$ showed higher expression level in the 5B- genotype, whereas the transcript was not detected in the genotype 5B+ (Table 3, Fig. 4). The other 16 high-confidence genes showed no differential expression levels between homozygous 5B+ and 5Bgenotypes. Resequencing of the parents revealed SNPs in the promoter region of TraesCS5B02G044800. Thus, TraesCS5B02G044800 was considered a candidate gene for QTgw.caas-5B.

\section{Validation of KASP markers flanking QTgw.caas-5B in a germplasm panel}

The Kasp_5B_Tgw based on the SNP with 'C' in ZM871 and 'A' in ZM895 alleles in the promoter region of TraesCS5B02G044800 for QTgw.caas-5B was used to genotype the diversity panel of 166 cultivars, among which 48 cultivars had the ZM895 genotype, and 118 had the ZM871 genotype (Table S3, Table 4). The ZM895 genotype showed significantly $(P<0.05)$ higher TGW and GL than the ZM871 genotype in all six environments as well as BLUE value. Differences in TGW and GL between the two genotypes ranged from 2.1 to $2.9 \mathrm{~g}$ (5.5 to $5.9 \%)$ and 0.30 to $0.40 \mathrm{~mm}$ (2.0 to $3.0 \%$ ), respectively (Table 4$)$. These results provided further evidence for a significant effect of QTgw.caas-5B on TGW.

\section{Discussion}

\section{Residual heterozygous recombinant lines are useful stocks for fine mapping}

Many QTL for grain-related traits have been identified in different genetic backgrounds (Huang et al. 2003; Quarrie et al. 2005; Prashant et al. 2012; Cui et al. 2014; Wu et al. 2015; Ma et al. 2018; Su et al. 2018; Zhai et al. 2018; Xu et al. 2019). Most were located in large chromosome intervals due to limited numbers of markers or lack of recombination events within the targeted QTL regions. Following release of the Chinese Spring reference genome sequence (IWGSC, https://urgi.versailles.inra.fr/blast_iwgsc/) and development of new sequencing technologies densely populated genetic maps can easily be constructed and genetic information for a specific map interval can be searched. Consequently, many researchers have employed fine mapping approaches to validate QTL or narrow genomic intervals within targeted QTL region (Röder et al. 2008; Brinton et al. 2017, Chen et al. 2020). In this study, progenies 12 heterozygous recombinant plants were genotyped and phenotyped to fine map QTgw.caas-5B to an approximately $2.0 \mathrm{Mb}$ physical interval containing 17 high-confidence annotated genes.

\section{Candidate genes for QTL controlling TGW}

The approximate $2.0 \mathrm{Mb}$ interval of QTgw.caas-5B was flanked by Kasp_5B29 and Kasp_5B31 (49.6-51.6 Mb). To identify candidate genes for TGW expression levels of the annotated were determined. TraesCS5B02G044800 showed as significantly lower expression level in the 5B+ genotype than in the 5B- genotype at all grain developmental stages. The ZM895 genotypes had significantly higher TGW and GL than ZM871 genotypes in the germplasm panel assessed with the Kasp_5B_Tgw marker based on a SNP developed from TraesCS5B02G044800, thus indicating that TraesCS5B02G044800 is a potential candidate gene for QTgw.caas-5B. Now, it is necessary to undertake gene overexpression and knockout studies to confirm the role of this gene candidate on TGW and GL. According to the Chinese Spring reference genome sequence (IWGSC, https://urgi.versailles.inra.fr/blast_iwgsc/), TraesCS5B02G044800 were predicted to encode for a TIR-NBS-LRR disease resistance protein and another unknown functional protein. 


\section{GL contributes to TGW at QTgW.caas-5B locus}

Various studies suggest that the early stage of grain length development is important in determining final grain weight in wheat (Hasan et al. 2011, Guo et al. 2015, Simmonds et al. 2016, Brinton et al. 2017, 2018). This study initially detected a clear difference in GL between $5 \mathrm{~B}+$ and $5 \mathrm{~B}$ - genotypes at $12 \mathrm{DPA}$, whereas a corresponding difference in TGW between 5B+ and 5B- genotypes was first observed at 25 DPA. This supported the contention that GL is a main factor contributing to grain weight (Brinton et al. 2017).

\section{Applications in wheat breeding}

Major stable QTL for yield-related traits with tightly linked markers are very important for molecular breeding. In this study, a QTL for TGW on chromosome 5B showed stable effects on TGW and GL cross environments. Its presence in 48 accessions among a panel of 166 indicated that it had been a selected target for grain weight (or even yield) in past breeding programs, and thus Kasp_5B_Tgw represents a future target for marker assisted selection to enhance grain size and weight. Moreover, the current results provide a basis for map-based cloning of the gene underlying the QTL.

\section{Declarations}

Acknowledgements The authors are grateful to Prof. R. A. McIntosh, Plant Breeding Institute, University of Sydney, for critical review of this manuscript. This work was funded by the CAAS Agricultural Science and Technology Innovation Program (CAAS-ZDRW202002) and the Core Research Budget of the Non-profit Governmental Research Institutions (S2021ZD04).

Author contribution DZ and LY performed the experiment and wrote the paper. DZ, LY, DL, JZ and JY participated in the field trials and trait evaluation. SC, XX and XS provided extensive revision of the manuscript. ZH and YZ designed the experiment and wrote the paper. All authors read the final version of the manuscript and approved its publication.

\section{Compliance with ethical standards}

Conflicts of interest All authors declare that they have no conflicts of interest.

Ethical standards We declare that these experiments complied with the ethical standards in China.

\section{References}

1. Brinton J, Simmonds J, Minter F, Leverington-Waite M, Snape J, Uauy C (2017) Increased pericarp cell length underlies a major quantitative trait locus for grain weight in hexaploid wheat. New Phytol 215:1026-1038

2. Brinton J, Simmonds J, Uauy C (2018) Ubiquitin-related genes are differentially expressed in isogenic lines contrasting for pericarp cell size and grain weight in hexaploid wheat. BMC Plant Biol 18:22

3. Cabral AL, Jordan MC, Larson G, Somers DJ, Humphreys DG, McCartney CA (2018) Relationship between QTL for grain shape, grain weight, test weight, milling yield, and plant height in the spring wheat cross RL4452/'AC Domain'. PLoS One 13:e0190681

4. Cao S, Xu D, Hanif M, Xia X, He Z (2020) Genetic architecture underpinning yield component traits in wheat. Theor Appl Genet 133:1811-1823

5. Chen Z, Cheng X, Chai L, Wang Z, Bian R, Li J, Zhao A, Xin M, Guo W, Hu Z, Peng H, Yao Y, Sun Q, Ni Z (2020) Dissection of genetic factors underlying grain size and fine mapping of QTgw.cau-7D in common wheat (Triticum aestivum L.). Theor Appl Genet 133:149-162

6. Cui F, Zhao C, Ding A, Li J, Wang L, Li X, Bao Y, Li J, Wang H (2014) Construction of an integrative linkage map and QTL mapping of grain yield-related traits using three related wheat RIL populations. Theor Appl Genet 127:659-675

7. Dong L, Wang F, Liu T, Dong Z, Li A, Jing R, Mao L, Li Y, Liu X, Zhang K, Wang D (2014) Natural variation of TaGASR7-A1 affects grain length in common wheat under multiple cultivation conditions. Mol Breeding 34:937-947

8. Fischer RA, Edmeades GO (2010) Breeding and cereal yield progress. Crop Sci 50:85-98

9. Gao F, Ma D, Yin G, Rasheed A, Dong Y, Xiao Y, Xia X, Wu X, He Z (2017) Genetic progress in grain yield and physiological traits in Chinese wheat cultivars of Southern Yellow and Huai Valley since 1950. Crop Sci 57:760-773 
10. Guan P, Di N, Mu Q, Shen X, Wang Y, Wang X, Yu K, Song W, Chen Y, Xin M, Hu Z, Guo W, Yao Y, Ni Z, Sun Q, Peng H (2019) Use of near-isogenic lines to precisely map and validate a major QTL for grain weight on chromosome 4AL in bread wheat (Triticum aestivum L.). Theor Appl Genet 132:2367-2379

11. Guan P, Lu L, Jia L, Kabir MR, Zhang J, Lan T, Zhao Y, Xin M, Hu Z, Yao Y, Ni Z, Sun Q, Peng H (2018) Global QTL analysis identifies genomic regions on chromosomes $4 \mathrm{~A}$ and $4 \mathrm{~B}$ harboring stable loci for yield-related traits across different environments in wheat (Triticum aestivum L.). Front Plant Sci 9:529

12. Guo Z, Chen D, Schnurbusch T (2015) Variance components, heritability and correlation analysis of anther and ovary size during the floral development of bread wheat. J Exp Bot 66:3099-3111

13. Hanif M, Gao F, Liu J, Wen W, Zhang Y, Rasheed A, Xia X, He Z, Cao S (2015) TaTGW6-A1, an ortholog of rice TGW6, is associated with grain weight and yield in bread wheat. Mol Breeding 36:1

14. Hasan AK, Herrera J, Lizana C, Calderini DF (2011) Carpel weight, grain length and stabilized grain water content are physiological drivers of grain weight determination of wheat. Field Crops Res 123:241-247

15. Hu M-J, Zhang H-P, Cao J-J, Zhu X-F, Wang S-X, Jiang H, Wu ZY, Lu J, Chang C, Sun G-L, Ma C-X (2016) Characterization of an IAAglucose hydrolase gene TaTGW6 associated with grain weight in common wheat (Triticum aestivum L.). Mol Breeding $36: 25$

16. Huang XQ, Cöster H, Ganal MW, Röder MS (2003) Advanced backcross QTL analysis for the identification of quantitative trait loci alleles from wild relatives of wheat (Triticum aestivum L.). Theor Appl Genet 106:1379-1389

17. Jiang Y, Jiang Q, Hao C, Hou J, Wang L, Zhang H, Zhang S, Chen X, Zhang X (2015) A yield-associated gene TaCWI, in wheat: its function, selection and evolution in global breeding revealed by haplotype analysis. Theor Appl Genet 128:131-143

18. Kuchel H, Williams KJ, Langridge P, Eagles HA, Jefferies SP (2007) Genetic dissection of grain yield in bread wheat. I. QTL analysis. Theor Appl Genet 115:1029-1041

19. Langridge $P$ (2013) Wheat genomics and the ambitious targets for future wheat production. Genome 56:545-547

20. Li F, Wen W, Liu J, Zhang Y, Cao S, He Z, Rasheed A, Jin H, Zhang C, Yan J, Zhang P, Wan Y, Xian X (2019a) Genetic architecture of grain yield in bread wheat based on genome-wide association studies. BMC Plant Biol 19:168

21. Li N, Li Y (2016) Signaling pathways of seed size control in plants. Curr Opin Plant Biol 33:23-32

22. Li N, Xu R, Li Y (2019b) Molecular networks of seed size control in plants. Annu Rev Plant Biol 70:435-463

23. Ma F, Xu Y, Ma Z, Li L, An D (2018) Genome-wide association and validation of key loci for yield-related traits in wheat founder parent Xiaoyan 6. Mol Breeding 38:91

24. Ma L, Li T, Hao C, Wang Y, Chen X, Zhang X (2016) TaGS5-3A, a grain size gene selected during wheat improvement for larger kernel and yield. Plant Biotechnol J 14:1269-1280

25. Prashant R, Kadoo N, Desale C, Kore P, Dhaliwal HS, Chhuneja P, Gupta V (2012) Kernel morphometric traits in hexaploid wheat (Triticum aestivum L.) are modulated by intricate QTL $\times$ QTL and genotype $\times$ environment interactions. J Cereal Sci 56:432-439

26. Quarrie SA, Steed A, Calestani C, Semikhodskii A, Lebreton C, Chinoy C, Steele N, Pljevljakusic D, Waterman E, Weyen J, Schondelmaier J, Habash DZ, Farmer P, Saker L, Clarkson DT, Abugalieva A, Yessimbekova M, Turuspekov Y, Abugalieva S, Tuberosa R, Sanguineti MC, Hollington PA, Aragues R, Royo A, Dodig D (2005) A high-density genetic map of hexaploid wheat (Triticum aestivum L.) from the cross Chinese Spring $\times$ SQ1 and its use to compare QTLs for grain yield across a range of environments. Theor Appl Genet 110:865-880

27. Röder MS, Huang XQ, Borner A (2008) Fine mapping of the region on wheat chromosome 7D controlling grain weight. Funct Integr Genomics 8:79-86

28. Sajjad M, Ma X, Habibullah Khan S, Shoaib M, Song Y, Yang W, Zhang A, Liu D (2017) TaFlo2-A1, an ortholog of rice Flo2, is associated with thousand grain weight in bread wheat (Triticum aestivum L.). BMC Plant Biol 17:164

29. Simmonds J, Scott P, Leverington-Waite M, Turner AS, Brinton J, Korzun V, Snape J, Uauy C (2014) Identification and independent validation of a stable yield and thousand grain weight QTL on chromosome 6A of hexaploid wheat (Triticum aestivum L.). BMC Plant Biol 14:191

30. Simmonds J, Scott P, Brinton J, Mestre TC, Bush M, Del Blanco A, Dubcovsky J, Uauy C (2016) A splice acceptor site mutation in TaGW2-A1 increases thousand grain weight in tetraploid and hexaploid wheat through wider and longer grains. Theor Appl Genet 129:1099-1112 
31. Su Q, Zhang X, Zhang W, Zhang N, Song L, Liu L, Xue X, Liu G, Liu J, Meng D, Zhi L, Ji J, Zhao X, Yang C, Tong Y, Liu Z, Li J (2018) QTL detection for kernel size and weight in bread wheat (Triticum aestivum L.) using a high-density SNP and SSR-based linkage map. Front Plant Sci 9:1484

32. Su Z, Hao C, Wang L, Dong Y, Zhang X (2011) Identification and development of a functional marker of TaGW2 associated with grain weight in bread wheat (Triticum aestivum L.). Theor Appl Genet 122:211-223

33. Sun C, Zhang F, Yan X, Zhang X, Dong Z, Cui D, Chen F (2017) Genome-wide association study for 13 agronomic traits reveals distribution of superior alleles in bread wheat from the Yellow and Huai Valley of China. Plant Biotechnol J 15:953-969

34. Wang S, Yan X, Wang Y, Liu H, Cui D, Chen F (2016) Haplotypes of the TaGS5-A1 gene are associated with thousand-kernel weight in Chinese bread wheat. Front Plant Sci 7:783

35. Wang S, Zhang X, Chen F, Cui D (2015) A single-nucleotide polymorphism of TaGS5 gene revealed its association with kernel weight in Chinese bread wheat. Front Plant Sci 6:1166

36. Wu QH, Chen YX, Zhou SH, Fu L, Chen JJ, Xiao Y, Zhang D, Ouyang SH, Zhao XJ, Cui Y, Zhang DY, Liang Y, Wang ZZ, Xie JZ, Qin JX, Wang GX, Li DL, Huang YL, Yu MH, Lu P, Wang LL, Wang L, Wang H, Dang C, Li J, Zhang Y, Peng HR, Yuan CG, You MS, Sun QX, Wang JR, Wang LX, Luo MC, Han J, Liu ZY (2015) High-density genetic linkage map construction and QTL mapping of grain shape and size in the wheat population Yanda1817 x Beinong6. PLoS One 10:e0118144

37. Xu D, Wen W, Fu L, Li F, Li J, Xie L, Xia X, Ni Z, He Z, Cao S (2019) Genetic dissection of a major QTL for kernel weight spanning the Rht-B1 locus in bread wheat. Theor Appl Genet 132:3191-3200

38. Yang J, Zhou YJ, Wu QH, Chen YX, Zhang PP, Zhang YE, Hu WG, Wang XC, Zhao H, Dong LL, Han J, Liu Z, Cao TJ (2019) Molecular characterization of a novel TaGL3-5A allele and its association with grain length in wheat (Triticum aestivum L.). Theor Appl Genet 132:1799-1814

39. Yang L, Zhao D, Meng Z, Xu K, Yan J, Xia X, Cao S, Tian Y, He Z, Zhang Y (2020) QTL mapping for grain yield-related traits in bread wheat via SNP-based selective genotyping. Theor Appl Genet 133:857-872

40. Yue A, Li A, Mao X, Chang X, Li R, Jing R (2015) Identification and development of a functional marker from 6-SFT-A2 associated with grain weight in wheat. Mol Breeding 35:63

41. Zhai H, Feng Z, Du X, Song Y, Liu X, Qi Z, Song L, Li J, Li L, Peng H, Hu Z, Yao Y, Xin M, Xiao S, Sun Q, Ni Z (2018) A novel allele of TaGW2-A1 is located in a finely mapped QTL that increases grain weight but decreases grain number in wheat (Triticum aestivum L.). Theor Appl Genet 131:539-553

42. Zhang L, Zhao YL, Gao LF, Zhao GY, Zhou RH, Zhang BS, Jia JZ (2012) TaCKX6-D1, the ortholog of rice OsCKX2, is associated with grain weight in hexaploid wheat. New Phytol 195:574-584

43. Zhang Y, Liu J, Xia X, He Z (2014) TaGS-D1, an ortholog of rice OSGS3, is associated with grain weight and grain length in common wheat. Mol Breeding 34:1097-1107

\section{Tables}

Table 1 Mean thousand grain weights (TGW), grain lengths (GL) and grain widths (GW) of sets of 20 near-isogenic lines (NILs) with $5 \mathrm{~B}+$ and and 5B-from progenies of $\mathrm{L} 2925$ at different grain development stages 


\begin{tabular}{|c|c|c|c|c|c|c|c|c|}
\hline Trait & Genotype & $4 \mathrm{DPA}^{\mathrm{a}}$ & 8 DPA & 12 DPA & 16 DPA & 20 DPA & 25 DPA & 30 DPA \\
\hline \multirow[t]{2}{*}{ TGW (g) } & $\begin{array}{l}\text { 5B+ } \\
(\mathrm{ZM} 895)\end{array}$ & $2.1 \pm 0.1^{b} A^{c}$ & $4.8 \pm 0.1 \mathrm{~A}$ & $9.6 \pm 0.1 \mathrm{~A}$ & $16.0 \pm 0.3 \mathrm{~A}$ & $23.4 \pm 1.1 \mathrm{~A}$ & $36.6 \pm 0.2 \mathrm{~A}$ & $48.7 \pm 0.2 \mathrm{~A}$ \\
\hline & $\begin{array}{l}\text { 5B- } \\
(\mathrm{ZM} 871)\end{array}$ & $2.1 \pm 0.2 \mathrm{~A}$ & $4.7 \pm 0.2 \mathrm{~A}$ & $9.5 \pm 0.3 \mathrm{~A}$ & $15.2 \pm 0.3 \mathrm{~A}$ & $22.4 \pm 0.6 \mathrm{~A}$ & $35.2 \pm 0.2 B$ & $45.5 \pm 0.4 \mathrm{~B}$ \\
\hline \multirow[t]{2}{*}{$\mathrm{GL}(\mathrm{mm})$} & $\begin{array}{l}\text { 5B+ } \\
(\mathrm{ZM} 895)\end{array}$ & $3.82 \pm 0.11 \mathrm{~A}$ & $6.04 \pm 0.07 \mathrm{~A}$ & $7.85 \pm 0.05 \mathrm{~A}$ & $8.66 \pm 0.03 \mathrm{~A}$ & $8.46 \pm 0.03 \mathrm{~A}$ & $8.76 \pm 0.04 \mathrm{~A}$ & $8.50 \pm 0.03 \mathrm{~A}$ \\
\hline & $\begin{array}{l}\text { 5B- } \\
(\mathrm{ZM} 871)\end{array}$ & $3.83 \pm 0.11 \mathrm{~A}$ & $5.97 \pm 0.07 A$ & $7.71 \pm 0.04 \mathrm{~B}$ & $8.51 \pm 0.02 B$ & $8.35 \pm 0.04 \mathrm{~B}$ & $8.63 \pm 0.02 B$ & $8.37 \pm 0.05 B$ \\
\hline \multirow[t]{2}{*}{$\begin{array}{l}\text { GW } \\
(\mathrm{mm})\end{array}$} & $\begin{array}{l}\text { 5B+ } \\
(\mathrm{ZM} 895)\end{array}$ & $3.02 \pm 0.05 \mathrm{~A}$ & $3.51 \pm 0.03 \mathrm{~A}$ & $3.61 \pm 0.01 \mathrm{~A}$ & $4.09 \pm 0.03 \mathrm{~A}$ & $4.36 \pm 0.01 \mathrm{~A}$ & $4.73 \pm 0.04 \mathrm{~A}$ & $4.87 \pm 0.02 \mathrm{~A}$ \\
\hline & $\begin{array}{l}\text { 5B- } \\
(\mathrm{ZM} 871)\end{array}$ & $3.04 \pm 0.05 \mathrm{~A}$ & $3.50 \pm 0.04 \mathrm{~A}$ & $3.59 \pm 0.01 \mathrm{~A}$ & $4.05 \pm 0.04 \mathrm{~A}$ & $4.37 \pm 0.01 \mathrm{~A}$ & $4.72 \pm 0.02 \mathrm{~A}$ & $4.84 \pm 0.03 \mathrm{~A}$ \\
\hline
\end{tabular}

a DPA, days post-anthesis

${ }^{b}$ Data are means $\pm S D$

${ }^{\mathrm{C}}$ Phenotypic differences between contrasting genotypes followed by different letters are significant at $P<0.05$

Table 2 Mean thousand grain weight (TGW), grain length (GL) and grain width (GW) of near-isogenic lines (NILs) 5B+ and 5B-progeny from each recombinant line $(\mathrm{RL})$ 


\begin{tabular}{|c|c|c|c|c|c|}
\hline Line & Genotype & No. ${ }^{a}$ & TGW (g) & $\mathrm{GL}(\mathrm{mm})$ & $\mathrm{GW}(\mathrm{mm})$ \\
\hline \multirow[t]{3}{*}{ RL1 } & $5 B+(Z M 895)$ & 25 & $49.7 \pm 1.2^{b}$ & $6.88 \pm 0.05$ & $3.29 \pm 0.05$ \\
\hline & 5B- (ZM871) & 27 & $47.0 \pm 1.4$ & $6.78 \pm 0.07$ & $3.25 \pm 0.06$ \\
\hline & & & $2.7^{\star \star c}$ & $0.10 * \star \star$ & $0.04^{\star}$ \\
\hline \multirow[t]{3}{*}{ RL2 } & $5 B+(Z M 895)$ & 33 & $48.7 \pm 1.4$ & $6.84 \pm 0.05$ & $3.40 \pm 0.05$ \\
\hline & 5B- (ZM871) & 42 & $48.0 \pm 1.4$ & $6.77 \pm 0.05$ & $3.38 \pm 0.05$ \\
\hline & & & 0.7 & $0.07 * \star \star$ & 0.02 \\
\hline \multirow[t]{3}{*}{ RL3 } & $5 B+(Z M 895)$ & 24 & $48.5 \pm 1.0$ & $6.84 \pm 0.05$ & $3.38 \pm 0.03$ \\
\hline & 5B- (ZM871) & 32 & $48.2 \pm 2.1$ & $6.78 \pm 0.07$ & $3.39 \pm 0.06$ \\
\hline & & & 0.3 & $0.06 * \star$ & -0.01 \\
\hline \multirow[t]{3}{*}{ RL4 } & $5 B+(Z M 895)$ & 28 & $47.9 \pm 2.7$ & $6.89 \pm 0.10$ & $3.37 \pm 0.09$ \\
\hline & 5B- (ZM871) & 27 & $48.3 \pm 1.7$ & $6.84 \pm 0.06$ & $3.40 \pm 0.05$ \\
\hline & & & -0.4 & $0.05^{\star}$ & -0.03 \\
\hline \multirow[t]{3}{*}{ RL5 } & $5 B+(Z M 895)$ & 30 & $48.8 \pm 1.7$ & $6.82 \pm 0.11$ & $3.42 \pm 0.07$ \\
\hline & 5B- (ZM871) & 29 & $48.5 \pm 1.1$ & $6.80 \pm 0.15$ & $3.40 \pm 0.03$ \\
\hline & & & 0.03 & 0.02 & 0.02 \\
\hline \multirow[t]{3}{*}{ RL6 } & $5 B+(Z M 895)$ & 43 & $48.9 \pm 2.6$ & $6.87 \pm 0.10$ & $3.39 \pm 0.08$ \\
\hline & 5B- (ZM871) & 33 & $47.0 \pm 1.2$ & $6.80 \pm 0.09$ & $3.36 \pm 0.04$ \\
\hline & & & $1.9 * \star$ & $0.07 * \star \star$ & 0.03 \\
\hline \multirow[t]{3}{*}{ RL7 } & $5 B+(Z M 895)$ & 29 & $49.7 \pm 0.9$ & $6.98 \pm 0.06$ & $3.44 \pm 0.02$ \\
\hline & 5B- (ZM871) & 33 & $47.5 \pm 1.1$ & $6.88 \pm 0.07$ & $3.40 \pm 0.03$ \\
\hline & & & $2.2^{\star \star}$ & 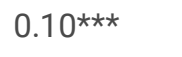 & $0.04^{\star}$ \\
\hline \multirow[t]{3}{*}{ RL8 } & $5 B+(Z M 895)$ & 33 & $48.6 \pm 2.1$ & $6.96 \pm 0.09$ & $3.40 \pm 0.07$ \\
\hline & 5B- (ZM871) & 31 & $48.5 \pm 2.0$ & $6.89 \pm 0.10$ & $3.41 \pm 0.06$ \\
\hline & & & 0.1 & $0.07 *$ & -0.01 \\
\hline \multirow[t]{3}{*}{ RL9 } & $5 B+(Z M 895)$ & 29 & $48.9 \pm 1.4$ & $6.90 \pm 0.08$ & $3.41 \pm 0.05$ \\
\hline & 5B- (ZM871) & 26 & $48.4 \pm 1.4$ & $6.84 \pm 0.09$ & $3.41 \pm 0.05$ \\
\hline & & & 0.5 & $0.06^{\star \star}$ & 0 \\
\hline \multirow[t]{3}{*}{ RL10 } & $5 B+(Z M 895)$ & 31 & $48.7 \pm 1.7$ & $6.95 \pm 0.08$ & $3.41 \pm 0.04$ \\
\hline & 5B- (ZM871) & 29 & $48.0 \pm 1.1$ & $6.86 \pm 0.09$ & $3.39 \pm 0.04$ \\
\hline & & & 0.7 & $0.09 \star \star \star$ & 0.02 \\
\hline \multirow[t]{3}{*}{ RL11 } & $5 B+(Z M 895)$ & 24 & $48.1 \pm 2.1$ & $6.93 \pm 0.09$ & $3.39 \pm 0.07$ \\
\hline & 5B- (ZM871) & 31 & $47.8 \pm 1.4$ & $6.86 \pm 0.07$ & $3.39 \pm 0.05$ \\
\hline & & & 0.3 & $0.07^{\star \star}$ & 0 \\
\hline RL12 & $5 B+(Z M 895)$ & 30 & $49.4 \pm 1.1$ & $6.88 \pm 0.02$ & $3.41 \pm 0.04$ \\
\hline
\end{tabular}

Page 10/15 


\begin{tabular}{|lllll|}
\hline $5 B-(\mathrm{ZM} 871)$ & 33 & $47.1 \pm 1.2$ & $6.80 \pm 0.07$ & $3.38 \pm 0.04$ \\
\hline & $2.3^{\star \star}$ & $0.08 * \star$ & 0.03 \\
\hline
\end{tabular}

a Number of lines within the corresponding genotypic group

${ }^{c}$ Data are means \pm SD

d Phenotypic difference between the means of genotypes 5B+ and 5B-. Asterisks indicate significant differences determined by ANOVA. * ** and ***, significant at $P<0.05, P<0.01$ and $P<0.001$, respectively

Table 3 Relative expression of TraesCS5B02G044800 in genotypes 5B+ and 5B- at different grain development stages

\begin{tabular}{|lllllll|}
\hline Genotype & 4 DPA $^{\mathrm{a}}$ & 8 DPA & $12 \mathrm{DPA}$ & $16 \mathrm{DPA}$ & $20 \mathrm{DPA}$ & $25 \mathrm{DPA}$ \\
\hline $5 \mathrm{~B}+(\mathrm{ZM} 895)$ & OB & OB & OB & OB & $0 \mathrm{~B}$ & $0 \mathrm{~B}$ \\
\hline $5 \mathrm{~B}-(\mathrm{ZM} 871)$ & $1.02 \pm 0.10^{\mathrm{b}} \mathrm{A}^{\mathrm{C}}$ & $1.11 \pm 0.06 \mathrm{~A}$ & $1.18 \pm 0.23 \mathrm{~A}$ & $0.56 \pm 0.18 \mathrm{~A}$ & $0.81 \pm 0.14 \mathrm{~A}$ & $0.82 \pm 0.34 \mathrm{~A}$ \\
\hline
\end{tabular}

a DPA, days post-anthesis

${ }^{\mathrm{b}}$ Data are means $\pm \mathrm{SD}$.

c Phenotypic differences between two genotypes followed by different letters are significant at $P<0.001$.

Table 4 Mean thousand grain weights (TGW), grain lengths (GL) and grain widths (GW) of genotypes 5B+ and 5B- in the germplasm panel of 166 wheat cultivars grown in six environments

\begin{tabular}{|c|c|c|c|c|c|c|c|c|c|}
\hline Trait & Genotype & No. ${ }^{a}$ & $E 4^{b}$ & E5 & E6 & E7 & E8 & E9 & BLUEC $^{\mathrm{c}}$ \\
\hline \multicolumn{10}{|c|}{ Kasp_5BTgw } \\
\hline \multirow[t]{3}{*}{$\begin{array}{l}\text { TGW } \\
(\mathrm{g})\end{array}$} & $\begin{array}{l}5 B+ \\
(Z M 895)\end{array}$ & 48 & $43.0 \pm 5.4^{d}$ & $43.9 \pm 5.0$ & $51.9 \pm 5.3$ & $47.7 \pm 6.2$ & $41.6 \pm 5.0$ & $41.3 \pm 5.0$ & $45.1 \pm 4.8$ \\
\hline & $\begin{array}{l}5 B- \\
(Z M 871)\end{array}$ & 118 & $40.4 \pm 4.9$ & $41.5 \pm 5.7$ & $49.0 \pm 5.4$ & $45.1 \pm 5.8$ & $38.5 \pm 4.2$ & $39.0 \pm 4.9$ & $42.4 \pm 4.8$ \\
\hline & & & $2.6^{\mathrm{e} \star \star}$ & $2.4^{*}$ & $2.9 * *$ & $2.6^{\star}$ & $3.1^{\star \star \star}$ & $2.3^{\star \star}$ & $2.7 * \star$ \\
\hline \multirow[t]{3}{*}{$\begin{array}{l}\text { GL } \\
(m m)^{f}\end{array}$} & $\begin{array}{l}5 B+ \\
(Z M 895)\end{array}$ & 48 & $13.80 \pm 0.74$ & $13.57 \pm 0.74$ & $15.03 \pm 0.74$ & $15.02 \pm 0.71$ & $14.39 \pm 0.68$ & $14.28 \pm 0.71$ & $14.49 \pm 0.69$ \\
\hline & $\begin{array}{l}5 \mathrm{~B}- \\
(\mathrm{ZM} 871)\end{array}$ & 118 & $13.40 \pm 0.67$ & $13.17 \pm 0.63$ & $14.73 \pm 0.68$ & $14.71 \pm 0.69$ & $14.05 \pm 0.67$ & $13.93 \pm 0.71$ & $14.15 \pm 0.64$ \\
\hline & & & $0.40 * \star$ & $0.40 * \star \star$ & $0.30 *$ & $0.31 *$ & $0.34 * \star$ & $0.35^{\star \star}$ & $0.34 * \star$ \\
\hline \multirow[t]{3}{*}{$\begin{array}{l}\text { GW } \\
(\mathrm{mm})^{f}\end{array}$} & $\begin{array}{l}\text { 5B+ } \\
(\mathrm{ZM} 895)\end{array}$ & 48 & $6.66 \pm 0.41$ & $6.59 \pm 0.32$ & $7.39 \pm 0.31$ & $7.22 \pm 0.36$ & $6.92 \pm 0.35$ & $6.80 \pm 0.35$ & $7.10 \pm 0.31$ \\
\hline & $\begin{array}{l}5 B- \\
(Z M 871)\end{array}$ & 118 & $6.59 \pm 0.34$ & $6.49 \pm 0.43$ & $7.30 \pm 0.36$ & $7.14 \pm 0.40$ & $6.77 \pm 0.34$ & $6.63 \pm 0.39$ & $7.00 \pm 0.34$ \\
\hline & & & 0.07 & 0.10 & 0.09 & 0.08 & $0.15^{\star \star}$ & $0.17^{*}$ & 0.10 \\
\hline
\end{tabular}

a Number of lines with corresponding genotype 
b E4 to E9, Anyang 2012-2013, Suixi 2012-2013, Anyang 2013-2014, Suixi 2013-2014, Suixi 2014-2015 and Shijiazhuang 2014-2015, respectively

${ }^{\mathrm{c}}$ BLUE: Best linear unbiased estimation

d Data are shown as means $\pm S D$

e Phenotypic differences between two genotype 5B+ and 5B-. Asterisks indicate significant differences determined by ANOVA. *, ** and $* \star *$, significant at $P<0.05, P<0.01$ and $P<0.001$, respectively

${ }^{f} \mathrm{GL}$ and $\mathrm{GW}$ are mean lengths and widths of 20 grains

\section{Figures}

a

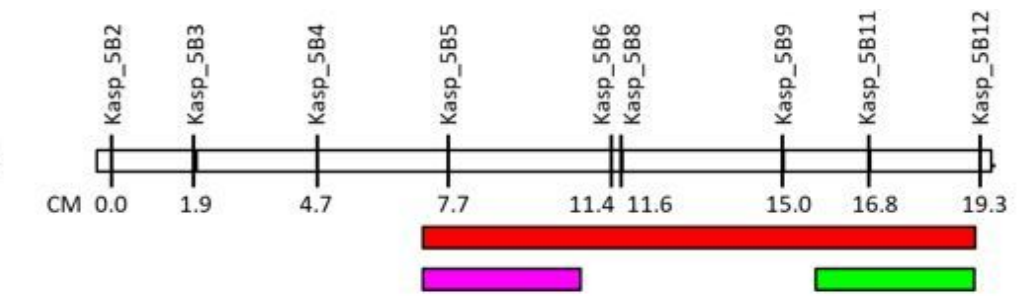

b

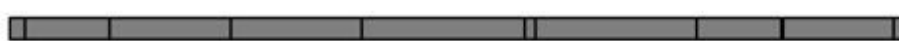

L2925
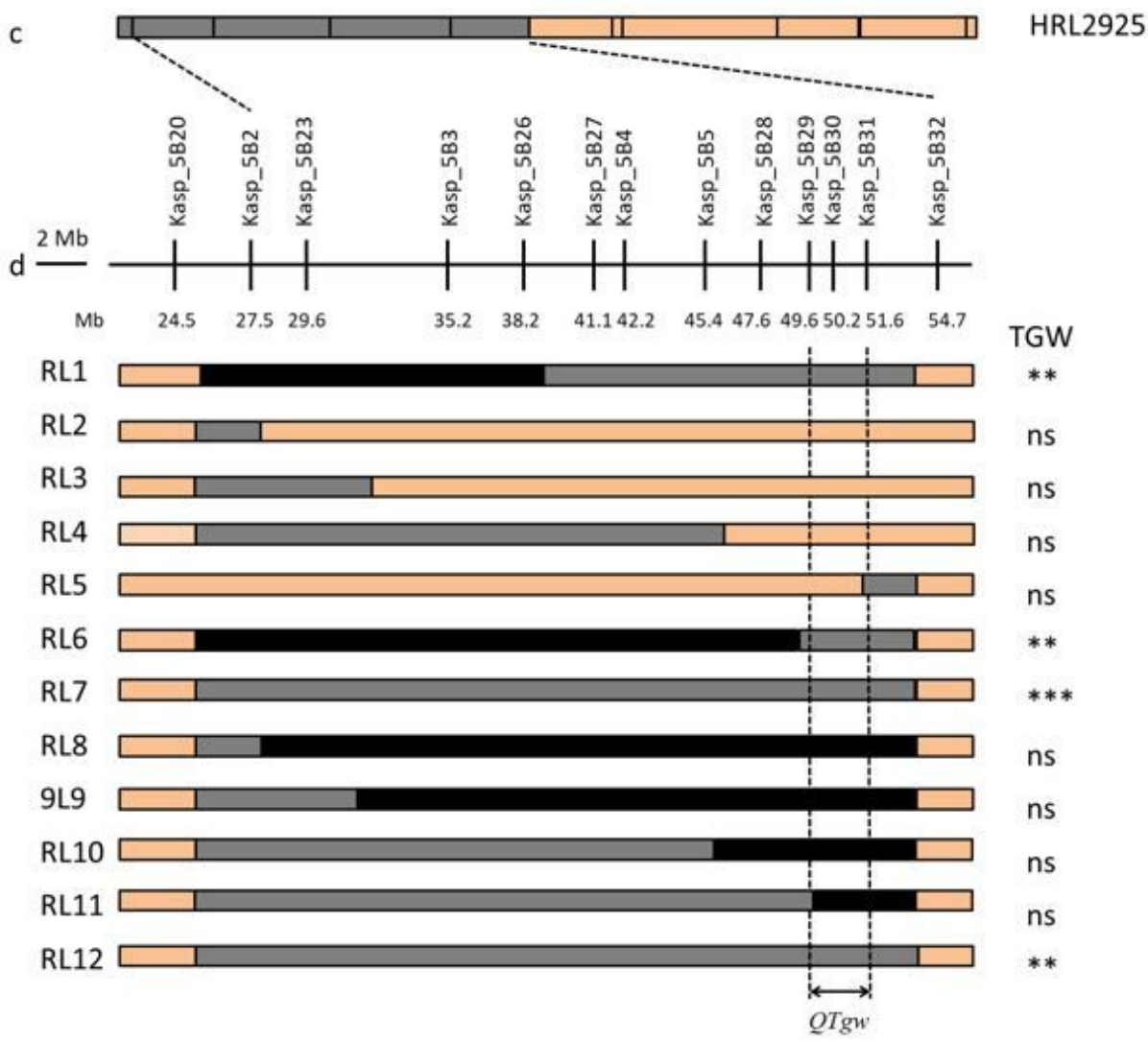

TGW $\square$ GL

$5 B+$

$\mathrm{GW}$

5B-

Heterozygous

Figure 1

Fine mapping of QTgw.caas-5B. a) Genetic map of 5B chromosome showing QTL for thousand grain weight (TGW), grain length (GL) and grain width (GW) in the Zhongmai 871/Zhongmai 895 RIL population from Yang et al (2020), b) Residual heterozygous line (L2925), c) Heterozygous recombinant plant (HRL2925), d) TGW map of 2.0 Mb interval on chromosome 5B. Left upside of 13 
markers used to screen recombinants are shown at the middle and graphical genotypes are shown for 12 recombinant line (RL1 to RL12). Statistical comparisons of TGW between $5 B+$ and 5B- genotypes among selfed progenies of each RL are shown at the right. *, $\star *, * * *$ and ns, significant at $P<0.05, P<0.01, P<0.001$ and non-significant, respectively

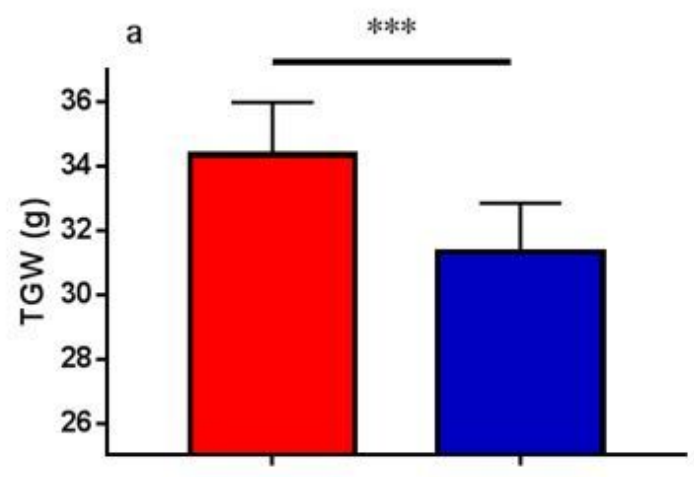

E1

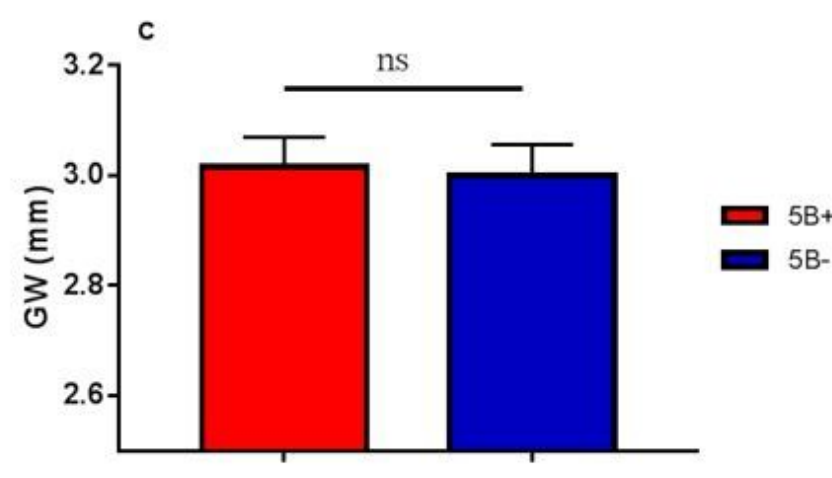

E1

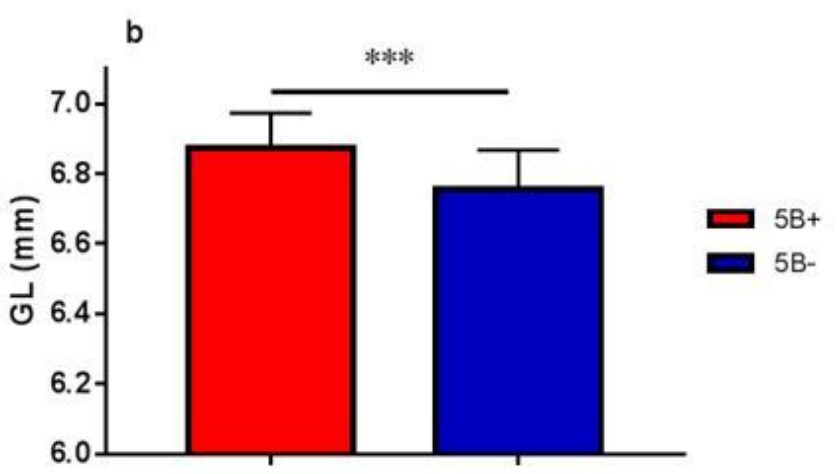

E1

\section{Figure 2}

Phenotypic differences in a) thousand grain weight (TGW), b) grain length (GL) and c) grain width (GW) between 5B+ and 5B-

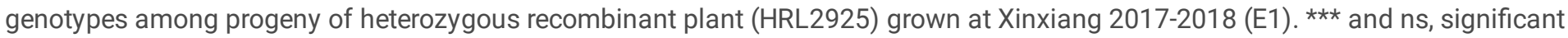
at $\mathrm{P}<0.001$ and non-significant, respectively 


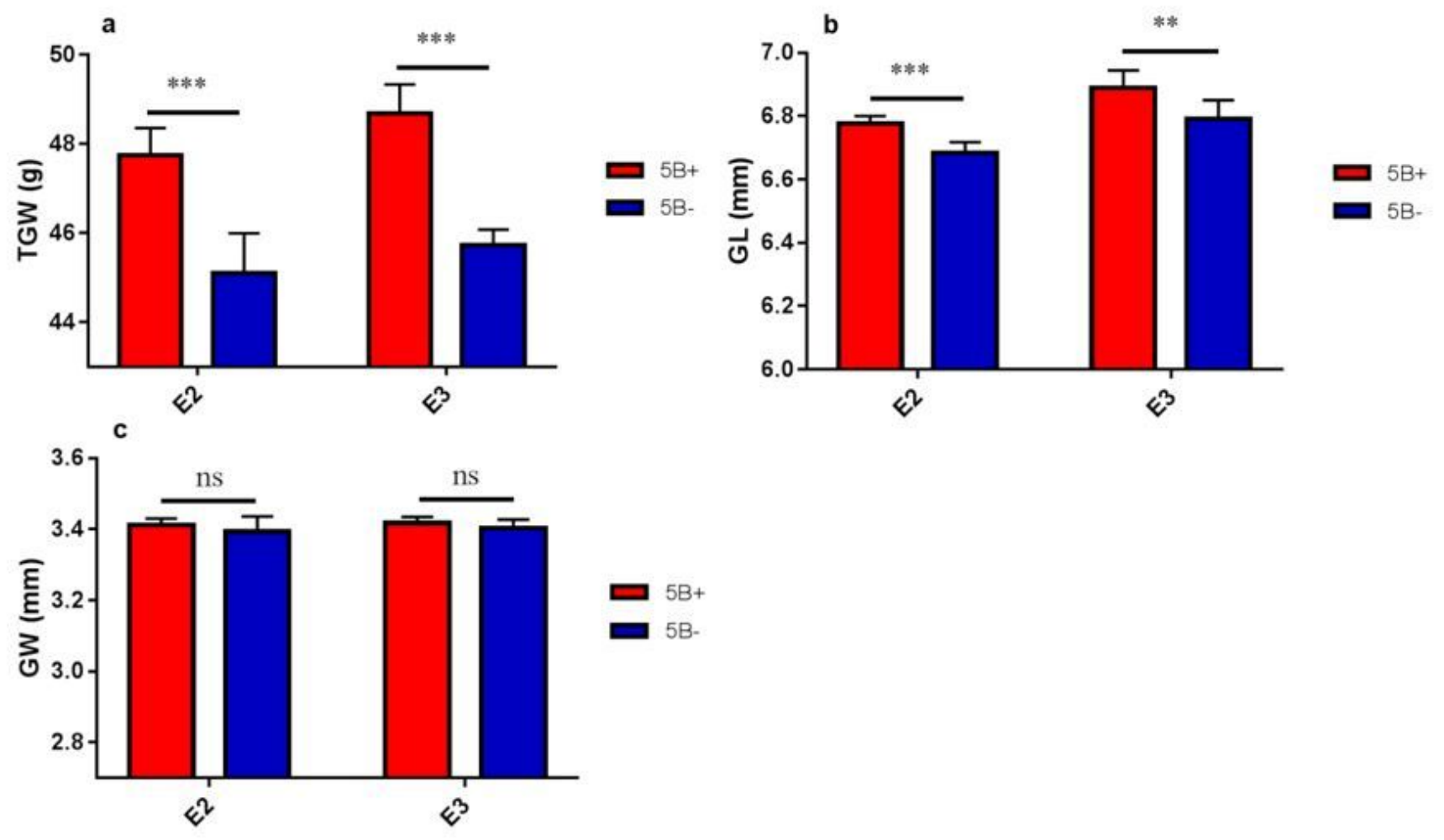

\section{Figure 3}

Phenotypic differences in a) thousand grain weight (TGW), b) grain length (GL) and c) grain width (GW) between 20 member sets of lines with genotypes 5B+ and5B-. E2 and E3, Anyang 2018-2019 and Xinxiang 2018-2019, respectively. ** $* \star \star$ and ns, significant at $P$ $<0.01, \mathrm{P}<0.001$ and non-significant, respectively 


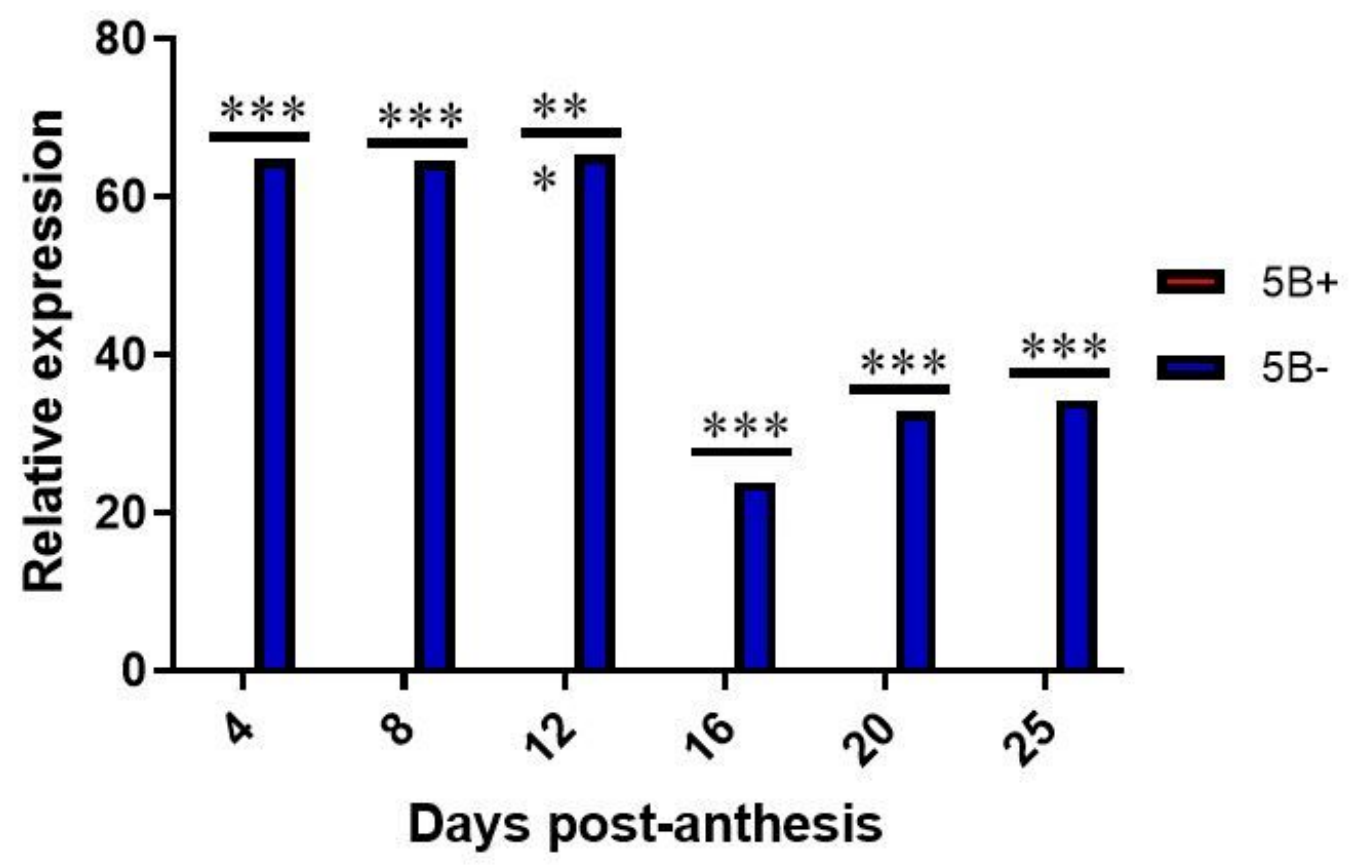

Figure 4

Relative expression of TraesCS5B02G044800 in 5B- and 5B+ genotypes at 4, 8, 12, 16, 20, 25 DPA (days post-anthesis). No transcript was detected in $5 \mathrm{~B}+$ genotypes. ${ }^{\star \star *}$, significant at $\mathrm{P}<0.001$

\section{Supplementary Files}

This is a list of supplementary files associated with this preprint. Click to download.

- SupplementrayTables.xlsx 